\title{
Spectroscopy of laser active media
}

\section{Thomas Glynn}

Thomas J. Glynn, "Spectroscopy of laser active media," Proc. SPIE 1603, Education in Optics, (1 March 1992); doi: 10.1117/12.57876

SPIE. Event: Education in Optics, 1991, Leningrad, Russian Federation 


\title{
Spectroscopy of laser active media.
}

\author{
T.J. Glynn. \\ National Centre for Laser Applications, \\ Physics Department, University College, \\ Galway, Ireland.
}

\begin{abstract}
Familiarity with the energy level structure in the active medium of a laser can help in understanding why particular pumping mechanisms are employed and how population inversion is achieved. Also, the allowed modes of operation (CW, pulsed, Q-switched, etc.) of a laser system are dictated by the excitation and decay kinetics of the excited states. Optical spectroscopic studies of the active media can provide insight into the operation of the different types of lasers and at the same time illustrate spectroscopic techniques as well as aspects of the energy level structure of solid, liquid, and gaseous media. A range of experiments and exercises are described illustrating concepts which are common to courses, both in optical spectroscopy and in the physics of lasers, and which could form a useful experimental option to such courses.
\end{abstract}

\section{INTRODUCTION}

Many undergraduate curricula in physics and engineering now incorporate courses on laser operation. The basic requirement for a laser is an active medium with an energy level structure which will allow population inversion to be obtained between a pair of levels ${ }^{1}$. This condition can be satisfied in many ways, reflected in the demonstration of laser action in a variety of laser media reported to date ${ }^{2,3}$. Only a small number of these systems have been developed into commercial products with particular applications in industry, communications, medicine, trade, etc. Experimental demonstrations of the basic spectroscopy of laser media can be a useful vehicle for illustrating many principles and techniques in spectroscopy as well as providing some insight into the limitations imposed by the energy level structure of the active medium on the spectral and temporal form of the laser output. With the development of new techniques in optical spectroscopy ${ }^{4}$ - tunable lasers, diode and CCD array detectors, microcomputer-based multichannel analysers, etc. - the equipment required for the experiments described below may be assembled from surplus items in many research establishments.

The experiments are of two types. In the first type the emission spectra of various laser active are recorded and since the emission is the basis of laser action the spectra are easy to observe with simple apparatus. In the second type of experiment the various aspects of the laser output are investigated. The experiments are usually carried out in the final undergraduate year after many of the topics investigated have been treated in a basic spectroscopy course.

\section{SOLID-STATE LASERS.}

Although it is not now widely used, the operation of the ruby laser is described in most laser textbooks since it marked the beginning of quantum electronics. As a result, it occupies a special position in relation to the study of laser physics and solidstate optical spectroscopy. The laser transition is between the lowest excited state $\left({ }^{2} \mathrm{E}\right)$ and the ground state $\left({ }^{4} \mathrm{~A}_{2}\right)$ of $\mathrm{Cr}^{3+}$ ions in $\mathrm{Al}_{2} \mathrm{O}_{3}$. The eigenfunctions describing these states are linear combinations of d-electron (even parity) wavefunctions and the transition is spin and parity-forbidden, but the selection rules are relaxed by admixtures of quartet states and higherlying odd states into both levels. As a result, the transition lifetime is quite long for an electric dipole transition ( $\sim 3 \mathrm{~ms}$ at room temperature). This lifetime can be measured using a filtered strobe source to excite the sample and a photomultiplier or photodiode (suitably filtered to block strobe light) to view the emission directly or through a small monochromator. The signal may be viewed on an oscilloscope or, preferably, using a multichannel scaler. If the latter is available, very weak sources such as a chopped high-radiance LED or He-Ne laser may be used for excitation. The $\mathrm{CW}$ emission can also be recorded to demonstrate the laser linewidth. Values for both the linewidth and the radiative lifetime are used in most textbooks to calculate the population inversion required at threshold for this system. Routine absorption measurements may be used to show the broad strong absorption bands of ruby which facilitate its successful pumping using flashlamps ${ }^{5}$. 

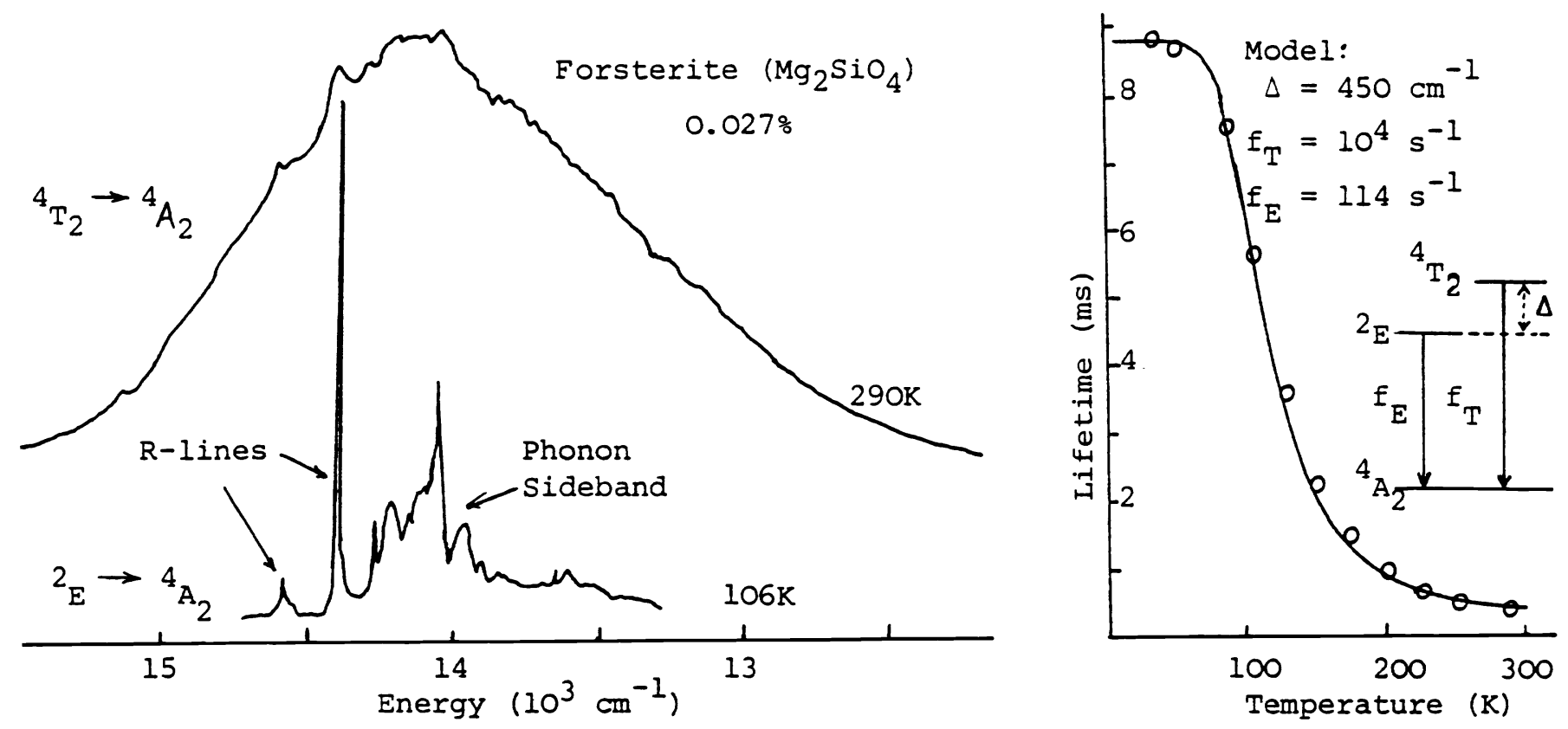

Fig. 1. (a) Luminescence spectrum of $\mathrm{Mg}_{2} \mathrm{SiO}_{4}: \mathrm{Cr}^{3+}$ at $106 \mathrm{~K}$ and $290 \mathrm{~K}$, showing the transition from ${ }^{2} \mathrm{E}$ emission to ${ }^{4} \mathrm{~T}_{2}$ emission. (b) Lifetime variation (measured at the $R_{1}$ wavelength) as a function of temperature.

Recently, other $\mathrm{Cr}^{3+}$-doped systems have been developed ${ }^{6}$ in which the ${ }^{2} \mathrm{E}-{ }^{4} \mathrm{~T}_{2}$ separation (Fig. 1(b)) is much smaller than in ruby. The ${ }^{2} \mathrm{E}$ level, being metastable, acts as a storage level for the ${ }^{4} \mathrm{~T}_{2}$ state with which it is in thermal equilibrium. The homogeneously-broadened ${ }^{4} \mathrm{~T}_{2} \rightarrow{ }^{4} \mathrm{~A}_{2}$ transition is spin-allowed and, in Cr-doped GSGG, it is the basis for tunable laser action over the range $740-840 \mathrm{~nm}$. The temperature-dependent luminescence and common lifetime of the excited states are easily recorded and show distinctly different behaviour at low temperature and at room temperature. Fig. 1(a) shows essentially the same behaviour occurring in $\mathrm{Cr}$-doped forsterite $\left(\mathrm{Mg}_{2} \mathrm{SiO}_{4}\right)$ although the reported laser action in this material is based on a different $\mathrm{Cr}$ center. The lifetime variation shown in Fig. 1(b) can be explained using a simple model ${ }^{7}$. Alternatively, having recorded the ruby spectra and lifetime, a dry-lab approach in which the analysis of supplied data (e.g. Fig. 1) and the extraction of important parameters (such as transition rates and energy separation ) is invited.

The simple emission spectrum of $\mathrm{Cr}^{3+}$-doped media, originating only on the lowest level, may be contrasted with that of Nd-doped systems, typified by Nd:YAG or Nd in glass (Fig. 2). Small pieces of these materials may be purchased from the manufacturers of laser rods. The lifetime of the laser transition is not so easily measured in this case as an S1 photomultiplier is required at $1.06 \mu \mathrm{m}$. However, a Ge photodiode may be used instead if available. Various sources may be used to excite the luminescence but it is particularly interesting to use a diode laser, operating at a wavelength corresponding to one of the narrow absorption bands of this medium, as the excitation source. This approach gives a nice demonstration of the operation of the compact diode-pumped Nd:YAG lasers which have been developed over the past 10 years. Fig. 2 shows the room temperature spectra of Nd-doped YAG crystal and ED-2 glass, both excited by a diode laser operating at $750 \mathrm{~nm}$. The absorption spectrum of the host can be used to show the best operating wavelengths for pumping Nd-doped media ${ }^{2}$.

The operation of the diode laser itself is the subject of a further experiment which begins with the study of the emission from semiconductor materials at various temperatures. LED devices are cheap sources of such materials, and information on the composition of the active media enclosed in these components may sometimes be available from the suppliers, or may be deduced by comparing the measured spectra with those published in the literature ${ }^{8-10}$. At low temperatures, emission from deep traps may be observed in indirect band-gap materials and this is replaced by the luminescence from shallower traps at room temperature. In direct band-gap materials, temperature-induced shifts of the band gaps may also be inferred from the band edge emission spectra at various temperatures $(77-300 \mathrm{~K})$. Finally, the emission spectrum of a diode laser as a function of drive current can be investigated. The transition from broad-band emission (as in the case of the LED) below threshold 


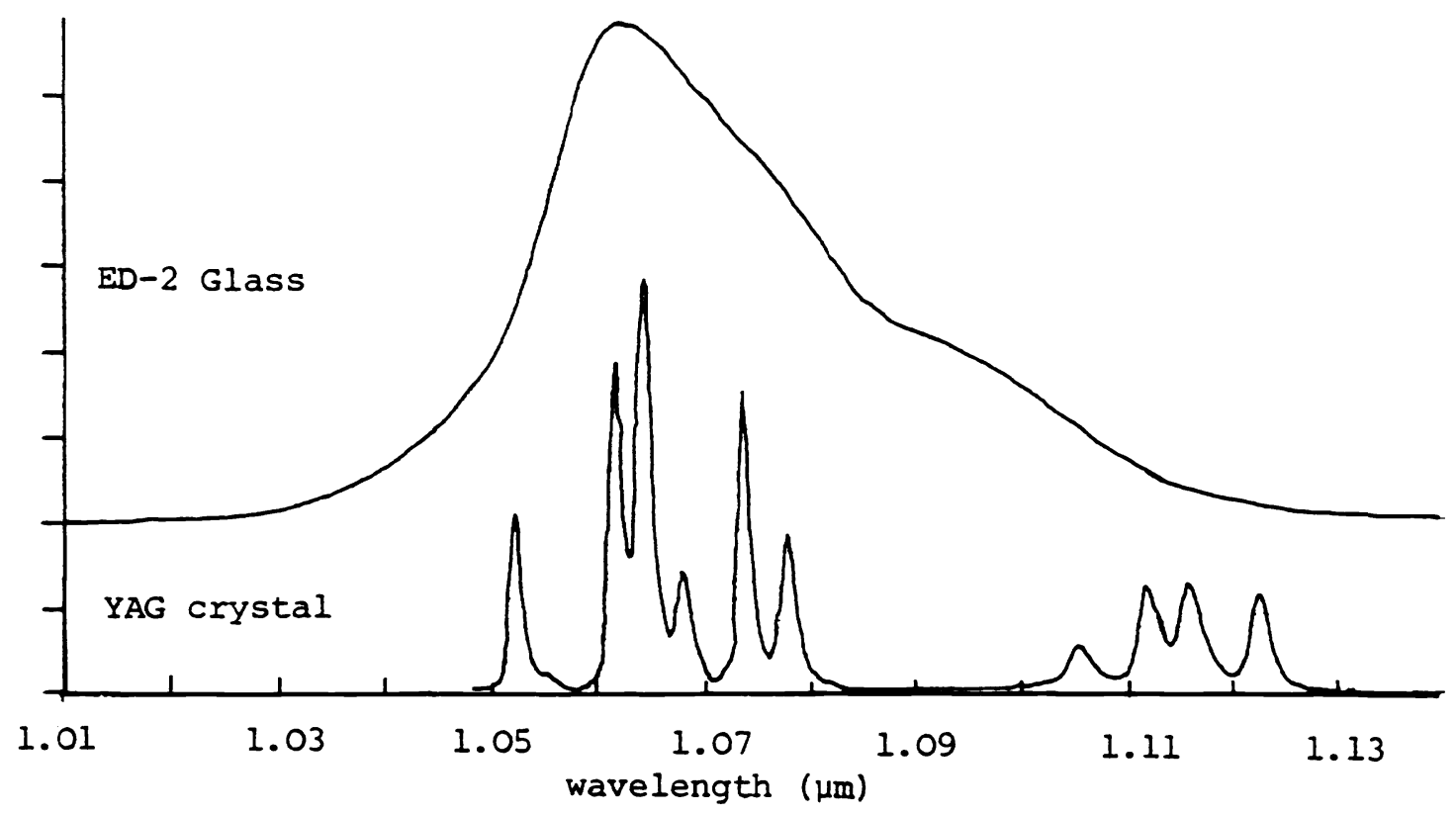

Fig. 2. Room-temperature emission in the region of the $1.06 \mu \mathrm{m}$ laser transition from Nd ions in YAG single crystal and in ED-2 laser glass, both excited by a diode laser operating at $750 \mathrm{~nm}$.

to the multi-longitudinal-mode spectrum above threshold is easily demonstrated. Using a power-meter, the output power/drive current characteristic can be plotted and the slope efficiency $(\eta)$ of the device can be measured. This parameter can then be related to the internal quantum efficiency of the laser transition. From the separation of the longitudinal modes, and a knowledge of the refractive index of the active medium, the length of the laser cavity can be calculated. The diode laser is now a very cheap device, finding applications in many areas, and many simple experiments can designed around it. Projects aimed at chopping the output for pulsed excitation studies or a digital communication setup can be used to extend the activities on this topic.

\section{GAS LASERS.}

The He-Ne laser is the simplest gas laser available, is cheap and easy to use, and already finds many uses in undergraduate teaching laboratories. The pumping scheme is described in detail in many textbooks but it is not generally mentioned in laser textbooks that the pumping efficiency for the laser transition on the $\mathrm{Ne}$ atoms is significantly enhanced by using $\mathrm{He}^{3}$ rather than $\mathrm{He}^{4}$ as the energy carrier in the discharge ${ }^{11}$. This feature enables an interesting measurement to be made on the isotope shift of spectral lines in the helium spectrum by comparing the emission from the He-Ne laser plasma with that from an ordinary helium discharge tube. The experimental arrangement is shown in Fig. 3. Light from both sources is directed onto the slits of a 1-metre spectrometer - the laser beam helps in aligning the plasma emission along the correct path - and the intensities in the lines due to the different isotopes are adjusted using filters or other means until they are comparable. A typical result for the $2^{1} \mathrm{~S}-3^{1} \mathrm{P}$ transition at $501.5 \mathrm{~nm}$ is also shown in Fig. 3. The measured shift is $0.861 \mathrm{~cm}^{-1}$, which is in good agreement with the theoretical prediction ${ }^{12}$ of $0.849 \mathrm{~cm}^{-1}$. An important feature of this experiment is that it will not be possible to resolve the isotope structure unless the 1-meter spectrometer (with $1200 \mathrm{~g} / \mathrm{mm}$ grating) is operated at almost maximum resolution. The effects of incomplete illumination of the grating, variation of slit width and height, etc, are explored in preliminary experiments. A quantitative calculation of the shift, based on a simple model for the energy levels, can be included for completeness or invited as part of the experimental account. Measurement of the shift leads to a realisation of the strong dependence of the energy transfer efficiency in atomic collisions on the energy mismatch.

The longitudinal mode spacing for the He-Ne laser can also be measured and compared with that measured for the diode laser shown above. For typical cavity lengths $(30 \mathrm{~cm})$, the longitudinal mode spacing is $\sim 500 \mathrm{MHz}$ which, combined with a Doppler gain profile of $1500 \mathrm{MHz}$, results in laser output in 2-3 longitudinal cavity modes. A Fabry-Perot etalon of moderate finesse and free spectral range can resolve this structure in the laser line. We have used a solid etalon (optical 

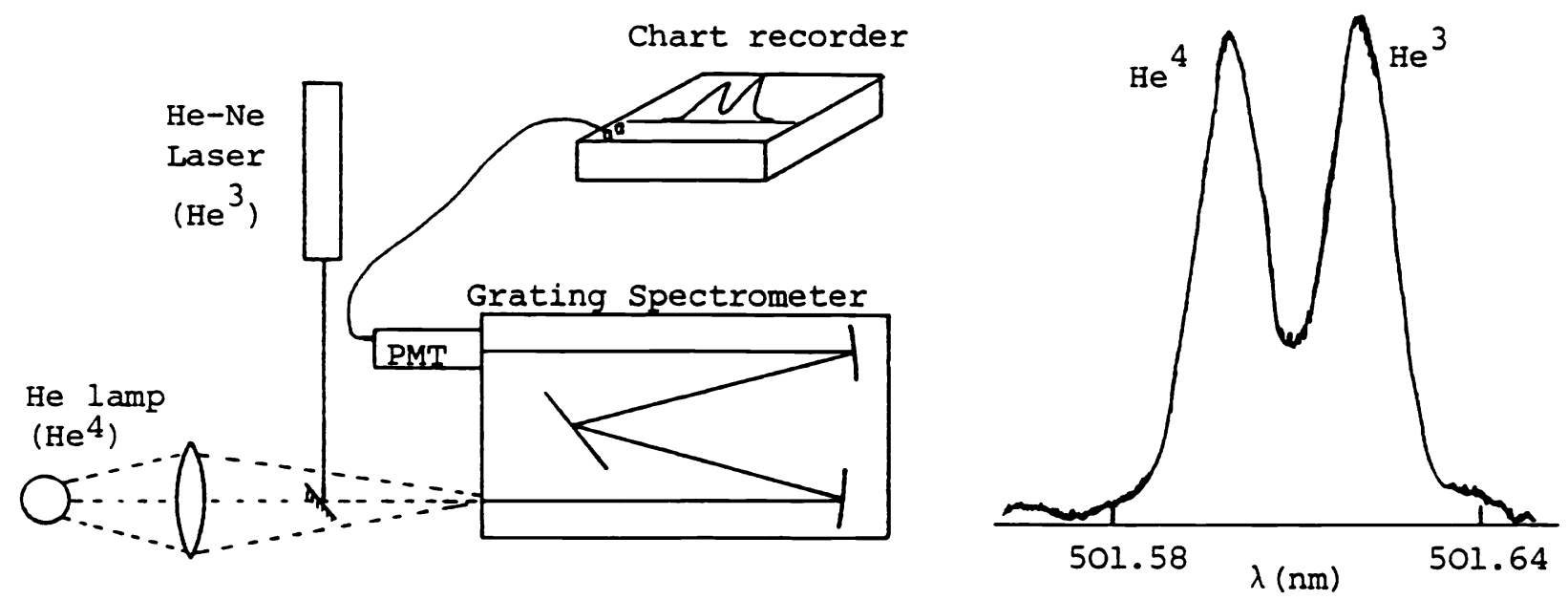

Fig. 3. Experimental arrangement used to observe the isotope shift in helium and a typical emission spectrum.

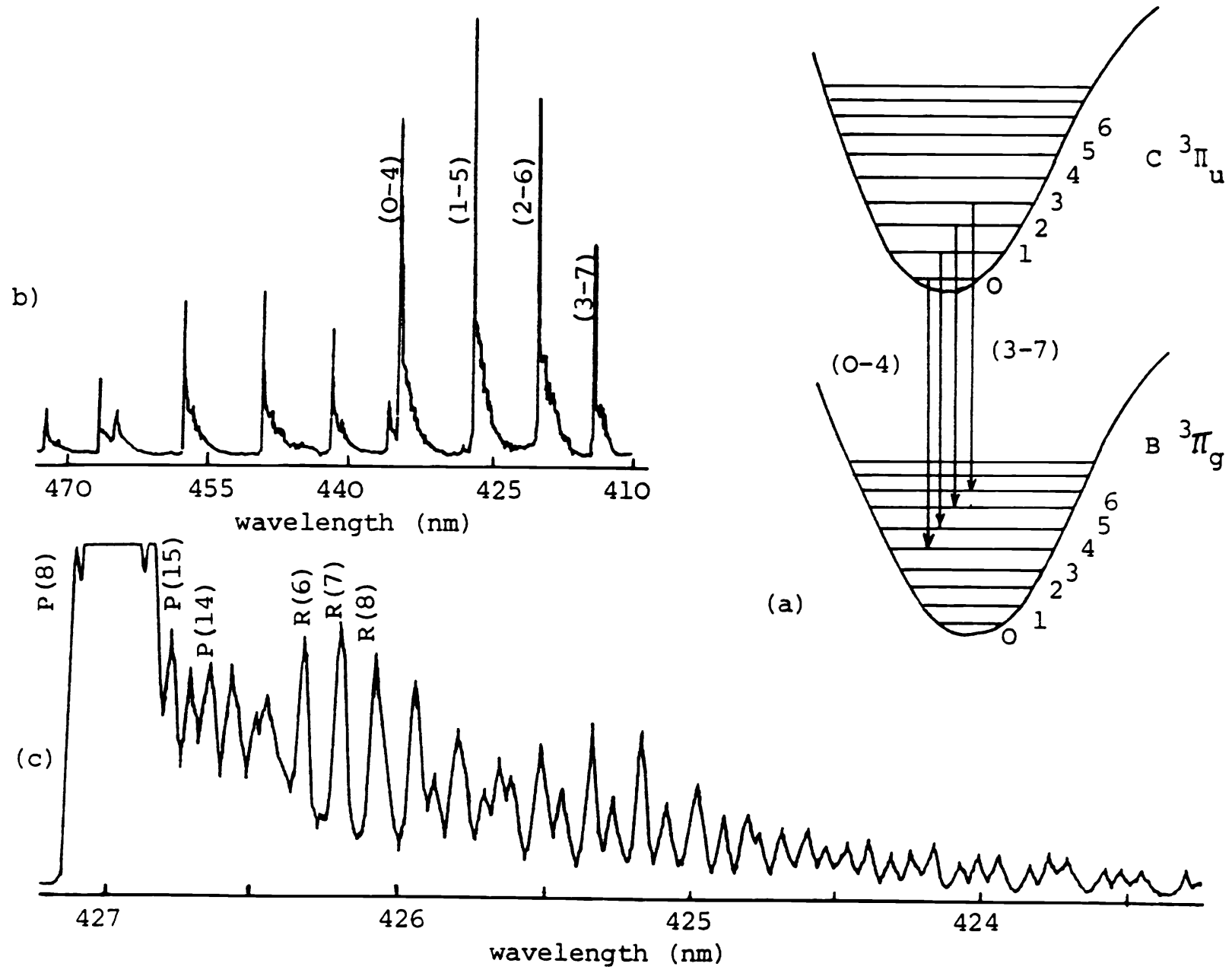

Fig. 4. (a) Part of the energy level diagram of $\mathrm{N}_{2}$, showing electronic and vibrational levels. The rotational fine structure is not shown. (b) Series of bands corresponding to the electronic transitions shown in (a). (c) The rotational fine structure in the $427 \mathrm{~nm}$ band shown in (b). Some transitions in the $R$ and $P$ branches are identified. The $P$ branch has turned round giving rise to the band "head" at the long wavelength end. 
thickness $1.5 \mathrm{~cm}$ ) with dielectric coatings giving a reflectivity finesse of $\sim 35$. This is useful when time is limited as no alignment of the F-P etalon is necessary. However, we have also used the F-P attachment on the Ealing-Beck interferometer which allows the plate separation to be varied. The ring pattern is photographed and from careful measurements of the ring diameters the laser cavity length can be calculated. As the laser cavity warms up, the movement of the "comb" of longitudinal cavity modes across the gain profile can be detected from the movement of the F-P rings in the pattern.

Following a simple design ${ }^{13}$, two home-made nitrogen lasers were constructed as student projects. The presentation of the energy level diagram for this laser system provided the stimulus to study the spectroscopy of molecular nitrogen displayed in a simple discharge. The origins of the electronic-vibrational-rotational transitions which give rise to emission in the UV and visible regions are described in many textbooks ${ }^{14}$. Using a 1-metre spectrometer, the R-branch and P-branch rotational structure can be resolved in any of the electronic-vibration bands as shown in Fig. 4. This transition is part of the 2nd positive band of $\mathrm{N}_{2}$, corresponding to transitions between the $\mathrm{C}^{3}{ }_{u}$ and $\mathrm{B}^{3}$ gelectronic levels. Laser action, which takes place on some 30 rotational transitions in a $0.1 \mathrm{~nm}$ interval at $337.1 \mathrm{~nm}$, corresponds to the $0-0$ band of this transition. In Fig. 4(c), the value of the rotational quantum number $\mathrm{J}$ for the initial state is given in parentheses. The J-value at which the P-branch turns around can be predicted from the tabulated values of the rotational constants in the upper and lower states $^{14}$. As an exercise, we suggest that the wavelengths of the various components in one of these bands can be predicted (once the transition is identified) from literature data and can be compared with the recorded spectrum to identify the rotational quantum numbers associated with individual components. Alternatively, by identifying the vibrational quantum numbers associated with the band heads, one can estimate the size of the vibrational quantum in the ground and excited states of molecular nitrogen.

\section{CONCLUSION.}

The experiments described above are a subset of those run in our department during the past 10 years. Many of the experiments were begun as extended projects aimed at developing the most efficient setup so that the experiment could become a set piece in subsequent years. However, many different approaches can be adopted in teaching this subject ${ }^{15}$ and the examples quoted do not constitute an exhaustive list. The topics covered in any institution will probably reflect the interests of the teaching staff as well as the range of spectroscopic insirumentation which is available.

\section{ACKNOWLEDGMENT}

The contributions of numerous undergraduate students to the work described above is gratefully acknowledged.

\section{REFERENCES}

1. J.T. Verdeyen, "Laser Electronics", Prentice-Hall International 1989

2. F.T. Arecchi and E.O. Schulz-Dubois, The Laser Handbook, Vol. 1, North-Holland, New York, 1972.

3. R.W. Engstrom, Photomultiplier Handbook, Published by RCA, pp. 155-157, 1980.

4. W. Demtroder, Laser Spectroscopy, Springer Verlag Series in Chemical Physics Vol. 5, Berlin, 1982.

5. W. Koechner, Solid-state Laser Engineering, Springer Verlag, New York, 1976.

6. B. Struve and G. Huber, "Laser performance of $\mathrm{Cr}^{3+}: \mathrm{Gd}(\mathrm{Sc}, \mathrm{Ga})$ garnet", J. Appl. Phys., Vol. 57, pp. 45-48, 1985.

7. T.J. Glynn, G.F. Imbusch, and G.W. Walker, "Luminescence from $\mathrm{Cr}^{3+}$ centers in forsterite", J. Lumin., Vol. 48/49, pp. 541-544, 1991.

8. R.Z. Bachrach, W.B. Joyce, and R.W. Dixon, "Optical-coupling efficiency of GaP:N green LED's", J. Appl. Phys., Vol. 44, pp. 5458-5462, 1973.

9. M.G. Craford, R.W. Shaw, A.H. Hersog, and W.O. Groves, "Radiative recombination mechanisms in GaAsP diodes with and without nitrogen doping" Jour. Appl. Phys, Vol. 43, pp. 4075-4083, 1972.

10. S.M. Sze, Physics of Semiconductor Devices, Wiley-Interscience, New York, Chapter 12, 1981.

11. S.George and M. Voelker, "Isotope shift in the spectrum of helium", Am. J. Phys. Vol. 48, 57-58, 1980.

12. I.I. Sobel'man, Introduction to the Theory of Atomic Spectra, Pergamon, New York, p. 227, 1972.

13. R.C. Hilborn, "An inexpensive, reliable, high-power molecular nitrogen laser." Am. J. Phys. Vol. 44, pp. 1172-1176, 1976.

14. G. Herzberg, Spectra of Diatomic Molecules, Van Nostrand, New York, 1950.

15. D.C. O'Shea and D.C. Peckham, "Resource Letter L-1 : Lasers", Am. J. Phys., Vol. 49, pp. 915-925, 1981. 